\title{
TenniVis: Visualization for Tennis Match Analysis
}

\author{
Tom Polk, Member, IEEE, Jing Yang, Yueqi Hu, and Ye Zhao
}

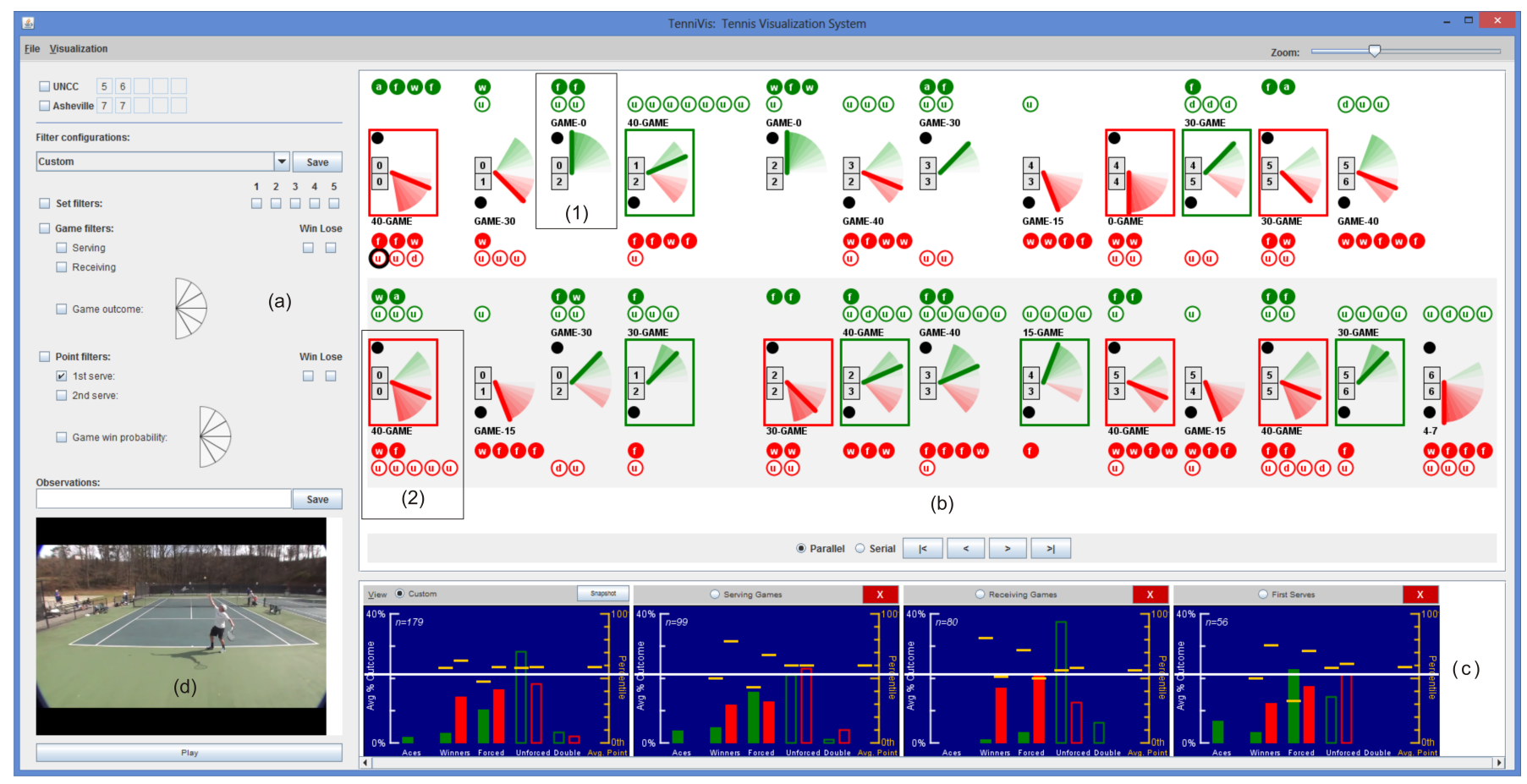

Fig. 1. A college men's singles tennis match in TenniVis. (a) Match scores and filters. (b) Pie Meter view. Each Pie Meter represents a game. Balls above/below it represent points gained by player one/two. Solid/hollow balls represent good (ace, winner, forced error) /bad (double-fault, unforced error) points. The darker the green/red colors in the pie, the better the chance player one/two had to win the game. Needle color shows who won the game (green for player one, red for player two). Needle angle indicates the final score (the closer to East, the closer the score was). Red and green boxes identify service breaks. (1) A game won easily by player one. (2) A game won with difficulty by player two. (c) Bar charts of point outcome statistics in multiple filter configurations. (d) Video viewer playing a video clip of a point.

\begin{abstract}
Existing research efforts into tennis visualization have primarily focused on using ball and player tracking data to enhance professional tennis broadcasts and to aid coaches in helping their students. Gathering and analyzing this data typically requires the use of an array of synchronized cameras, which are expensive for non-professional tennis matches. In this paper, we propose TenniVis, a novel tennis match visualization system that relies entirely on data that can be easily collected, such as score, point outcomes, point lengths, service information, and match videos that can be captured by one consumer-level camera. It provides two new visualizations to allow tennis coaches and players to quickly gain insights into match performance. It also provides rich interactions to support ad hoc hypothesis development and testing. We first demonstrate the usefulness of the system by analyzing the 2007 Australian Open men's singles final. We then validate its usability by two pilot user studies where two college tennis coaches analyzed the matches of their own players. The results indicate that useful insights can quickly be discovered and ad hoc hypotheses based on these insights can conveniently be tested through linked match videos.
\end{abstract}

Index Terms-Visual knowledge discovery, sports analytics, tennis visualization

\section{INTRODUCTION}

Visualization has been an important means of match analysis in a variety of sports $[11,12,14,18,19]$. For example, Perin et al. demonstrated the usefulness of visualizations in helping tactics analysts find insights into soccer games they would not have otherwise been able to

- Tom Polk, Jing Yang, and Yueqi Hu are with the University of North Carolina at Charlotte. E-mail: tepolk, jyang13,yhu12@uncc.edu.

- Ye Zhao is with Kent State University. E-mail: zhao@cs.kent.edu.

Manuscript received 31 March 2014.

For information on obtaining reprints of this article, please send e-mailto:tvcg@computer.org. find [18]. For tennis, much of the existing work utilizes ball and player tracking information to provide virtual replays of tennis matches [20]. This tracking information is also used to provide summary level information about player strategies such as player movement [21, 28]. A major challenge facing these approaches is that collecting the data typically requires an array of cameras and a system capable of handling the large amounts of video input in real time. While this might be reasonable for major, professional tennis tournaments, such a system is prohibitively expensive and impractical for the large body of non-professional tennis players at the college, high-school, and club levels.

Meanwhile, comparatively little work has been done on visualization of non-spatial data of tennis matches, such as score, point outcomes, point lengths and service information. Such data is easy to 

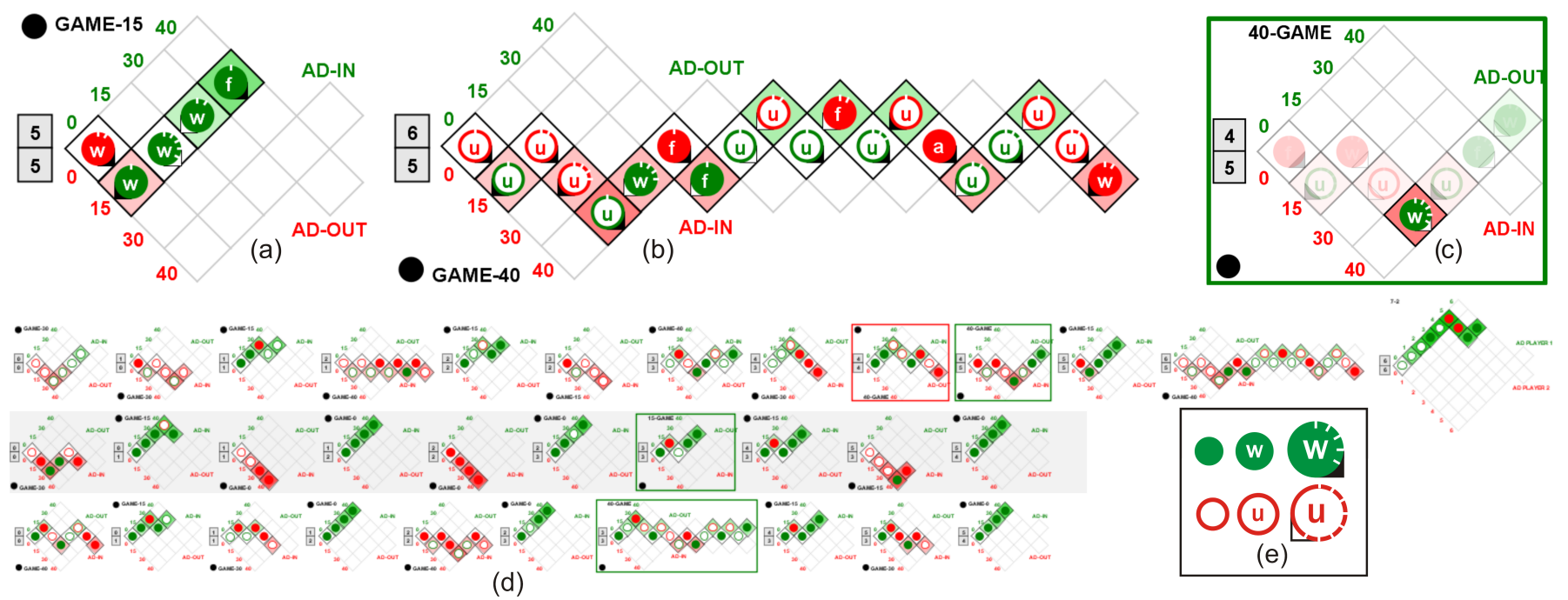

Fig. 2. Fish Grid views of the Federer-Gonzalez match (won by Federer 7-6, 6-4, 6-4). (a)-(c) are captured from a zoomed-in view and (d) is a zoomed out view. Each Fish Grid is a score matrix rotated 45 degrees with point outcomes mapped on it as balls (green/red: point gained by Federer/Gonzalez; solid/hollow: good/bad shot). The horizontal order of the balls indicates the temporal order of the points. The vertical position of a ball indicate who was in lead before the point (above the horizontal, Federer is in the lead; below the horizontal, Gonzalez is in the lead). (a) A game won easily by Federer. (b) The longest game in the match (won by Gonzalez). (c) A game with a "choke" point for Gonzalez, identified using filters. (d) Fish Grids for the entire match. Although shown small here, they still convey trend information for each game. (e) Sample Point Outcome Glyphs (balls) at different zoom levels. At the maximum zoom level, tick marks represent point length in seconds and triangles represent service side (left/right) and first vs. second serve (solid/hollow).

collect by non-professional players and carries both high level summaries and low level details about a match. Unfortunately, this data is usually analyzed in aggregated statistics and thus valuable insights about local details and trends are often missing.

The above observation inspired us to build a tennis visualization system for non-professional players based on non-spatial data. We thus develop a new system with the following goals:

- To be economically and technologically feasible for nonprofessional tennis players. In other words, no expensive ball and player tracking equipment and analysis software will be required.

- To provide easy to learn visualizations and easy to use interactions. Therefore, tennis coaches and players will be able to use the system without the accompaniment of visualization experts.

- To support users quickly discovering patterns about players and matches; to facilitate them in ad hoc hypothesis generation and evaluation.

- To allow coaches and players to easily share insights gained from the visualizations.

Our new system, named TenniVis, provides the following features to meet the above goals:

- Easy-to-collect data input: TenniVis merely requires the following information as its inputs: (1) the match video captured by a single consumer-level video camera and (2) non-spatial data that can be easily collected by someone spectating a match (i.e., parent, coach, or teammate) in real time. It includes timestamps marking the start and end of each point, indication of second serve, and point outcome (i.e., ace, double-fault, winner, forced error, or unforced error). Other information, including score information, who is serving, service side (ad or deuce court), game length (i.e., number of points), point length, and service breaks, can be derived from the data and the inherent structure of a tennis match.
- Easily learnable visualizations: TenniVis provides two novel views that can be quickly learned and understood by novice users: the Pie Meter view and the Fish Grid view. The Pie Meter view (see Figure 1 (b)) provides a high-level overview of an entire match. It reveals who won a game and the degree to which the score in the game fluctuated in favor of one player. The Fish Grid view (see Figure 2) provides more details at the game level by displaying each point outcome as the game score progresses. The game length, trend, and critical points can be easily observed from this view.

- Ad hoc hypothesis generation and evaluation: A variety of player, set, game, and point level filters are provided, allowing users to generate hypotheses about point outcomes. Users can evaluate these hypotheses using the outcome bar chart snapshots (see Figure 1 (c)) as well as by reviewing the video associated with specific points.

- Result sharing: Users can annotate individual points with their observations from the associated video clips (see Figure 1 (d)) These points are highlighted in the visualizations for easy review. The annotated video clips are automatically assembled into an HTML report that can easily be shared with players or coaches.

Non-professional tennis players and coaches have participated and provided important input to the development of TenniVis. First, the first author, who is also the developer of the system, has over 35 years of tennis playing experience. Second, a set of face-to-face meetings, phone interviews, and user studies have been conducted with nonprofessional tennis coaches. The coaches confirmed the feasibility of data collection for TenniVis. They also confirmed the usefulness of TenniVis in two pilot user studies where matches of their own players were analyzed.

The rest of the paper is organized as follows: Section 2 provides background knowledge on tennis and discusses challenges facing non-professional players when analyzing tennis matches. Section 3 presents related work. Section 4 presents TenniVis and illustrates its features using the case of a professional tennis match between Roger Federer and Fernando Gonzalez from the 2007 Australian Open Finals, won by Federer in three sets (7-6, 6-4, 6-4). Section 5 presents 
two pilot user studies of college tennis matches, where the analysis was performed by the coaches themselves. Section 6 presents our conclusions and future work.

\section{BACKground ANd REQUIREMENTS ANALYSIS}

In this section, we introduce background knowledge of tennis, point out match information that is important for match analysis, and highlight the requirements it brings to tennis visualization systems in bold fonts. The important information and requirements were identified according to the first author's own experience and intensive phone and face-to-face discussions with four club and college coaches.

Tennis lends itself to analysis due to its structured, hierarchical nature. A typical non-professional tennis match consists of the best two out of three sets, where each set is awarded to the player who gets six games first (with at least a two game margin). Each game is awarded to the player who gets four points first (with at least a two point margin). One player serves all the points in a game. Players alternate serving each game. They also change sides of the court after the first game and then after every two games. Serving is considered an advantage in tennis, so it is important to highlight games where the serving player loses the game. This is called a service break. Who is serving and service breaks should be immediately noticeable to users. If the game score within a set reaches six-six, a 12-point tiebreaker is played. In this tiebreaker one player serves one point and then each player takes turns serving two points. The player to get to seven points first with a margin of at least two points wins the tiebreaker and is awarded the set. If players get to six-six in the tiebreaker, they continue playing until one player gets a two point lead.

The scoring progression in a game is as follows: 0 (or love), 15, 30,40 , game. If, in a game, both players each get three points (called deuce), then play continues until one of the players gets a two point lead over the other player, thus winning the game. After a deuce point has been reached, when a player gets a point we say that player has the advantage. The score is usually reported from the server's viewpoint. In terms of risk, not all points within a game are equal. For example, a player serving at 40-0 in a game needs only to win one of the next three points to win the game. From the server's perspective, this is a far more comfortable position to be in relative to a score of 0-40 (known as a break point). Visually encoding the risks associated with the points will help users understand the pressure on the players at each point.

As with many sports, there is a psychological component to tennis that affects players differently. Some players ease off in their intensity after getting a lead just to see their lead slipping away (known as choking). Other players are able to step up and recover after being down in a match (known as rallying back). Choking and rallying back should be easy to spot in visualizations because they may identify player patterns that need to be modified.

A tennis point begins with a serve by one of the players. The starting location of the serve is behind the baseline on either the right half of the court (called the deuce side) or the left half (called the ad side). See [2] for an explanation of the various parts of a tennis court. If the server misses the first serve (i.e., it does not land in the correct service box), this is called a fault and the player gets to try again. If the player misses the second serve, this is called a double-fault and the point is awarded to the receiving player.

When analyzing a tennis match, information about whether a point started off on a first versus a second serve is very important because a player's second serve is usually not as powerful or effective as their first serve (thereby giving the receiving player an easier opportunity to return the serve). If the server hits the ball in the opponent's service box and the opponent does not touch the ball, this is called an ace and the point is awarded to the serving player.

Tennis points can end one of three additional ways besides by double-fault or ace: winner, forced error, or unforced error. A winner is when a player hits a legal shot (other than a serve) to his or her opponent and the opponent does not touch the ball. A forced error is similar to a winner except that the opponent is able to touch the ball. Both winners and forced errors (as well as aces) are "good" shots at- tributed to the "good" shot made by a player. An unforced error is when a player, despite having enough time to get to the ball and execute a good shot, ends up instead hitting the ball out of bounds or into the net. An unforced error, like a double-fault, is considered a "bad" shot attributed to the player that made the error because it gives a point to the opponent.

In analyzing tennis matches, understanding how points end is vitally important to understanding why a player is winning or losing his or her matches. The coaches that we talked to during our pilot user studies indicated that unforced errors help them identify potential shots a player needs to work on. They also indicated that winners made by an opposing player are often the result of poor shot selection on previous shots by a player. Thus, examining winners helps identify areas where strategies may need to be improved. Visually presenting point information, such as service information, good or bad shots, and point outcome types, can provide useful insights to coaches and players.

In addition to having information about the outcome of a point (ace, winner, unforced error etc.) and the service information (first versus second serve), the score of a game at which a specific outcome occurs is also important. For example, some players may have a tendency to start off games slowly and get behind. Seeing this in the visualization, coaches can work with a player to change their tactics or teach them how to be more mentally prepared at the start of a game. Therefore, it is very useful for visualization systems to visually present the point outcomes within the context of the score where they occur.

While video analysis is fairly common in many sports, such as American football, this is not as common in tennis matches, due primarily to the expense of analyzing this data. Unlike team sports where there is one match in which all members of the team participate, team tennis matches involve multiple, separate matches being played simultaneously. In NCAA [6] tennis matches, teams play three doubles matches followed by six singles matches. A typical singles match may last two or more hours, leading to 12 hours of video (for just singles) to be analyzed per team match. Many teams play 2 or 3 matches per week, resulting in 36 hours of video per week. It is time consuming for the coaches to analyze such a large volume of video. Meanwhile, it is important for coaches and players to watch the video of interesting points. Thus there is a dire need for visualization systems that can help coaches (or the players themselves) quickly identify points in a match that may lead to insights and watch the video clips associated with them, without having to sift through all of the raw video data.

\section{Related Work}

Many tennis visualization systems have been developed for enhancing commercial broadcasts of professional tennis matches at major tournaments or improving player performance. Most of them require ball and player tracking information. Jin and Banks [13] visualize tennis shots as two-dimensional ball traces and use a magic-lens to allow users to filter interesting points and to step through all points in a match through a time-varying display. However, they relied on a synthesized data set rather than on any actual ball trajectories. Systems such as HawkEye [17], LucentVision [22] and Tennissense [9, 10] require the use of an array of synchronized cameras to collect ball and player tracking information. HawkEye [17] and LucentVision [22] provide player movement data and ball landing positions for serves and shots during each point. This enables insights such as patterns, strengths, and weakness of players to be gained from the tracking data. These insights are provided to professional tennis analysts to enhance live broadcasts and are also used for novel visualizations for tennis fans, including the capability for fans to experience serves from the player's perspective. Tennissense $[9,10]$ automatically extracts key events such as games, change of ends, and serves from match videos. It presents a coaching interface where users can quickly jump to any event in a match to examine it from the perspective of any of the nine arrayed camera angles. The drawback of the above approaches is that they are prohibitively expensive and impractical for most nonprofessional tennis players. In addition, they can only be used for 

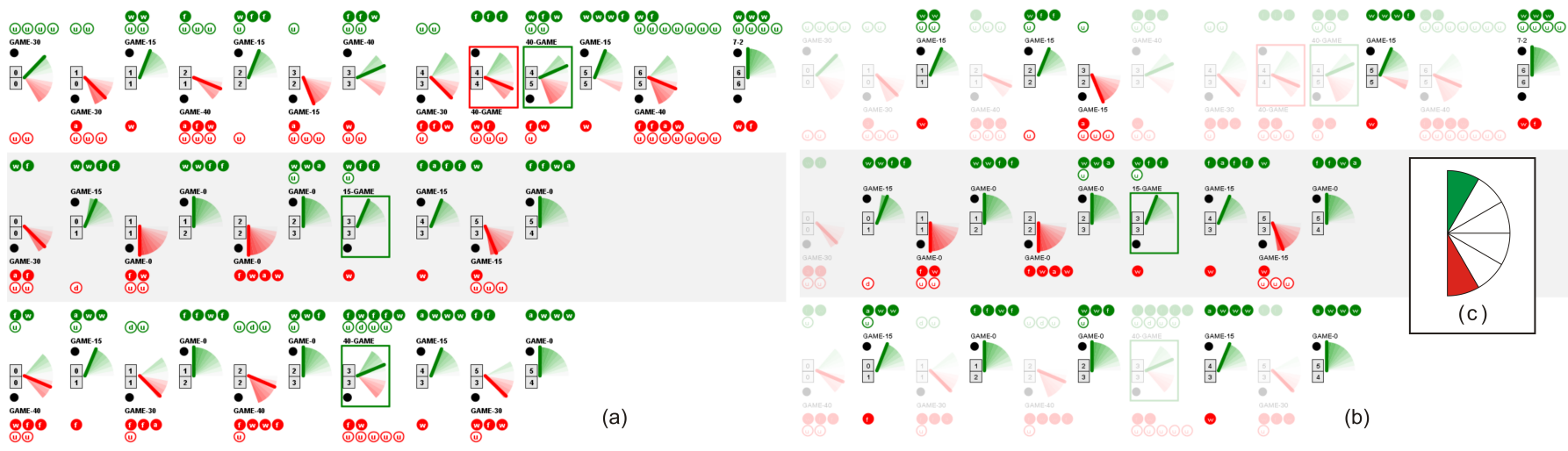

Fig. 3. Pie Meter view of the Federer-Gonzalez match. (a) Before filtering. All games are displayed. (b) After filtering. Only games won easily by either player are displayed. (c) The Game Outcome Filter used for the filtering.

matches played at the courts where the equipment is installed.

To avoid using the expensive equipment, several commercial products rely on human beings to collect the track information. They fall into two basic categories: motion analysis software (e.g., Dartfish [1], MotionView [3]) and tennis match point tracking (e.g., ProTracker [4]). The motion analysis software is not designed for analyzing entire matches, but rather is used for reviewing swing and service techniques. Players can capture tennis swings on high-speed cameras and then review it in slow-motion. They can also select objects in subsequent frames to show a swing path and they can add voiceovers to capture their observations. ProTracker [4] requires a third party (i.e., coach or parent of a player) to manually enter shot position information for serves, returns, and key shots. It then generates a report with match summary statistics, scatter diagrams for serves, returns, and key shots, and a momentum chart that identifies potential turning points in a match. These approaches require tedious human efforts to optionally enter location information manually for a small subset of the overall points (i.e., just serves and key shots).

Statistics have been widely used in analysis of tennis and other sports. Websites such as IBM's SlamTracker [5] present basic statistics from professional tennis matches, including the number of aces, double faults, winners, and unforced errors. They also present statistics such as the first and second serve win percentages and the percent of times a player wins when he or she approaches the net. TenniVis greatly leverages the power of statistics by interactive visualization: it allows users to flexibly select points of interest and generate bar charts to examine their aggregated statistics. Meanwhile, the users can immediately review the video of any points in the selection. In this way, they can immediately test any hypotheses inspired by the statistics.

Several visualizations and interactions in TenniVis, such as Point Outcome Glyphs, semantic zooming, and filtering, are inspired by previous work. Glyphs $[8,25,26]$, a traditional multidimensional visualization technique, have been used in sport visualization systems. For example, MatchPad [14] uses glyphs to encode event records in rugby. Semantic zooming, an interaction technique that couples zooming with changes in levels-of-detail, have been used in tree visualization [23], software visualization [16] and many other visualizations. Our pie-shaped filter is inspired by dynamic queries [7, 27].

\section{TenniVis}

\subsection{System Overview}

In order to address the information needs discussed in Section 2, TenniVis employs visualizations that mimic real-life metaphors, such as dial meters, to make it easy for coaches and players to learn and interpret the visualizations. Consistent visual encoding schemes are used across the visualizations to facilitate learning and allow for easy transition between visualizations.

Figure 1 shows the main screen of TenniVis. On the left are a set of filters (top) and a video viewer (bottom). On the right are the main visualization window (top) and an outcome bar chart panel (bottom) Users can switch between the Pie Meter view and the Fish Grid view in the main visualization window. The currently displayed visualization can be zoomed in or out using the zoom slider located above it. TenniVis provides a multi-resolution approach to allow users to examine a match at multiple levels of detail. In particular, the Pie Meter view (see Figure 1 (b)) provides a match level overview where users can quickly examine the outcome of all sets and games, see how intensive each game is, and effectively compare the performance of the two players.

The Fish Grid view (see Figure 2) allows users to examine the match in a finer level of detail. Users can quickly see the progression of points within a game, each point outcome (ace, winner, unforced error, etc.) against a backdrop of the current game score. They can also instantly distinguish between very short games where one player wins easily and very long games where the players battle back and forth.

The finest level of detail TenniVis provides is the playback of match video of a user-selected point. When a point is selected, the video clip for that point immediately begins playing in the video viewer (see Figure 1 (d)). The user can also open up a larger, secondary window to view a full screen version of the video (particularly useful in a multiple-monitor environment).

A rich set of interactions are provided in TenniVis using a spectrum of filters (see Figure 1 (a)) that, in combination, allow users to select semantically meaningful sets of points. This allows them to examine the percent distribution, point length, and first serve percentage of point outcomes (ace, winners, unforced errors, etc.) in bar charts. In this way, users can create ad hoc hypotheses on why specific games or points were won or lost. Since users can easily drill-down to any point to watch the corresponding video clip, these hypotheses can be conveniently tested. To share the insights captured in the visual exploration, users are able to make notes on their insights and to generate reports that consist of written text and video clips that can be shared with the player or coaches.

In the following sections, we introduce the data collection, visualizations and interactions of TenniVis in full detail. The match between Roger Federer and Fernando Gonzalez in the 2007 Australian Open Men's Singles Final (won by Federer 7-6, 6-4, 6-4) is used as an example dataset since many readers are familiar with these players.

\subsection{Data Collection}

Match videos are desired in tennis analysis since all insights ultimately need to be evaluated through them. As one of the tennis coaches in our pilot studies commented, "they (the players) need to see it". TenniVis allows users to rapidly identify points of interest and conveniently watch their video clips. Since ball and player tracking data is not used in TenniVis, only one consumer-level video camera is needed for video capturing and no tedious manual tracking data collection is required. 
The non-spatial data is manually collected on-the-fly by a person spectating a match. In order to support point-based selection of specific video clips, the start time and the end time of each point as well as who won the point, the type of outcome (i.e., ace, double-fault, winner, forced error, or unforced error), and service faults (i.e. missed serves) are recorded. This approach, while requiring the attention of the data collector throughout the match, can be easily collected using either a smart phone or smart watch app. The spectator only needs to synchronize the start time of the data collection app with the camera (before or after the match) and click a few buttons when a point starts and ends. There is ample time between points to perform this task. The data collection app can keep track of score and who is serving based on the rigid structure of a tennis match. Only one video per match is required. The TenniVis system simply indexes into specific points in the video based on the synchronized timestamps collected.

Data for historical matches can be collected in a similar way, with the only difference being that the spectator watches the match videos rather than the live matches. We asked several coaches about the feasibility of the players themselves collecting this data during a match (using a smart watch app) and they indicated this is not realistic. They noted, however, that they often have players who are not playing in a particular match who would be able to collect this data. The rigid structure of a tennis match allows a great deal of derived data to be collected from the trivial data items collected manually, including information such as score, who is serving, service breaks, break point opportunities, and point lengths (which is a surrogate measure for the number of shots in a rally).

\subsection{Pie Meter View}

Figure 3 (a) displays the Pie Meter view of the match between Federer and Gonzalez. It provides an overview of the match and also can be animated to present the dynamic process of the match. The visualization consists of multiple blocks. Each block visually presents a game. It contains a needle gauge-like glyph called a Pie Meter and rows of balls representing points called Point Outcome Glyphs (see Section 4.4). Games of the same set are placed in the same row, sorted from left to right by play order. The sets are ordered from top to bottom by play order. The score panels on the left of each Pie Meter show the game score within the set just prior to that game. A black ball shown at the top left of the Pie Meter indicates player one is serving, while a black ball on the bottom left indicates player two is serving. To highlight service breaks, games with a service break are surrounded by a red box (player two broke the serve of player one) or a green box (player one broke player two's serve).

Please note that in TenniVis, information about player 1 is always positioned in the top half of the visualization graphics and information about player 2 is always positioned in the bottom half of the visualization graphics, as exemplified by positioning of the black ball indicating who is serving. Similarly, green is always used to represent something good for player one and red is used to represent something good for player two. The highlight of service breaks is an example of this scheme. These encoding themes will be seen again and again in the rest of the paper. They are used in TenniVis since (1) A versus B is the most important information in tennis, an A versus B style sport; (2) According to Mackinlay [15], position and color hue are the most relevant encodings for perceptual tasks with nominal data. Thus they should be used for the most important information; and (3) Consistent visual encoding facilitates learning.

The needle gauge metaphor is employed in the Pie Meter since the rotation of a needle on a gauge is a familiar and natural way to reveal how a game progresses in favor of player one or player two. In our initial design, a full gauge covering 180 degrees is displayed and a needle dynamically traverses within the gauge as the game progresses. The position and the background color of the needle indicate whether the game is currently at a score in favor of player one or player two. After the initial prototype was implemented, we soon realized that the area traversed by the needle in a game reveals interesting information such as whether a game was dominated by one player or swung back and forth. Therefore, we revised the design and removed the part of a

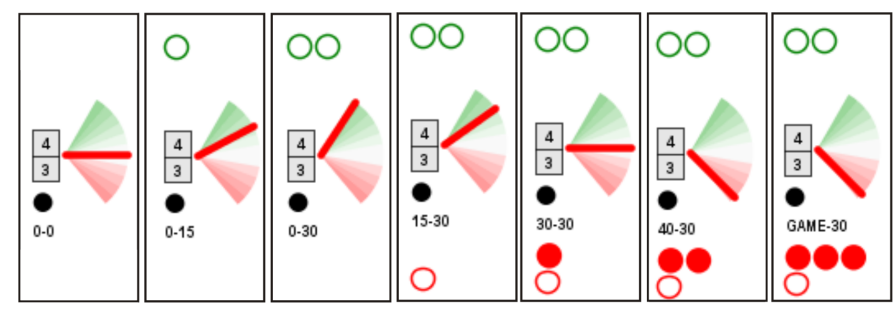

Fig. 4. Succession of points from left to right and their corresponding needle angle changes in the Pie Meter for a game. Needle color shows that player 2 won. The green gradient colors indicate advantage for player 1 and the red gradient colors indicate advantage for player 2 .

gauge not traversed by the needle in the game (so it looks like a pie). The details of the Pie Meter visual encoding is introduced below.

Following the color scheme, needle color shows who won the game (green for player one, red for player two). The gauge background (the pie) is divided into many sectors. Each of them is mapped to a range of conditional probabilities of player one winning the game (probabilities in short). The probabilities are encoded by colors of the sectors. Here again, green means player one has a better chance to win and red means player two has a better chance to win. The darker the color is, the higher the chance. The gauge is positioned vertically, with green sectors on the top half and red sectors on the bottom half.

All possible game scores are assigned a probability using a coinflip simulation, assuming that players are equally skilled. When a game progresses, the needle is placed in the gauge according to the probability of the current score. When the game score is equal, the needle is placed at a white sector oriented to the East. When the score is more favorable to player one/two, the needle moves to the green/red sectors, indicating player one/two has a bigger chance to win. After removing the sectors not traversed by the needle in a game, the display only consists of sectors covering all possibilities happening in the game. Therefore, users can judge the intensity of a game by the color and angles of its Pie Meter. As Figure 3 demonstrates, even when the Pie Meters are relatively small, games where one player dominated are easily distinguishable from close games that swung back and forth.

The above simplistic approach assumes that players are equally skilled, which, of course, is often not the case. Better players are able to come back and win a game, even when they are behind in the score. However, this color coding scheme still makes it easy to distinguish situations that are more favorable to a player winning a game (shades of green) from those that are less favorable (shades of red).

The Pie Meter can be used in a static mode or an animation mode. In the static mode, the needle angle indicates how close the final game score was. There are eight possible final game scores: Game-40, Game-30, Game-15, Game-0, 0-Game, 15-Game, 30-Game, and 40Game. These eight scores are mapped to needle angles ranging from +90 to -90 degrees (assuming East is considered 0 degrees). Once a game goes to deuce, the number of deuces is not considered: the score is mapped as Game-40. A similar approach is used for tie-break games.

A Pie Meter can be animated to step through the games (see Figure 4 for an example). When switching to the animation mode, all of the needles reset to a neutral, horizontal position (indicating neither player has an advantage). When player one wins a point, the needle angle moves up in a counter-clockwise direction and when player two wins a point, it moves down in a clockwise direction. Therefore, the angle of the needle indicates the current advantage (i.e., conditional probability of winning the game) one player has over the other and the final needle angle indicates how close the game was. In Figure 4 we can see that player one wins two points in a row, giving him a major advantage. Player two then rallies back, winning four points in a row to win the game.

Example 1 Figure 3 (a) reveals a few patterns about the match in 
which Federer (player one) won in straight sets (7-6, 6-4, 6-4). First, we see that Gonzalez (player two) broke Federer's serve first (indicated by the red box in the first set); but Federer broke right back. In sets two and three, there were only two service breaks (both by Federer) which gave him the match. The second pattern that emerges is that many of the games in the first and third sets involved swings from one player to another (indicated by Pie Meters with both red and green gradients displayed), while all games in set two were won fairly easily by one player or another.

\subsection{Point Outcome Glyphs}

According to the requirements analysis (see Section 2), the following information about point outcomes are desired, sorted by importance: (1) who won each point; (2) whether the outcome was due to a "good" shot by the player winning the point or a "bad" shot by her/his opponent; (3) the specific type of the point outcome; (4) at which score the point outcome was generated; (5) details about the point, such as how long the point was and service information. The Point Outcome Glyphs (the green and red balls in the Pie Meter and Fish Grids) are proposed to encode the above information for individual point outcomes.

Since there are many point outcomes in a game, the glyph representing an individual point outcome can't be too big. We select a ballshaped glyph design (see Figure 2 (e) for an example) since (1) it is easy for users to associate a ball with a point outcome; (2) it provides a maximum amount of interior space in which to write the outcome letter (i.e., 'a' for ace, 'w' for winner, etc.); and (3) it allows us to use the stopwatch metaphor to encode time as tick marks. To be consistent with our color scheme, green balls indicate points awarded to player one since they are good for player one. Red balls are for points awarded to player two since they are in favor of player two. In addition, solid balls represent good shots (aces, winners, and forced errors). Hollow balls represent bad shots (double faults and unforced errors). Following our positioning scheme, points awarded to player one were placed above the Pie Meters and points awarded to player two were placed beneath the Pie Meters. In the Fish Grid View, the balls are placed at the game score (e.g., 30-15) at which the point outcome occurred.

To allow users to examine the point outcome information at multiple levels of detail, the Point Outcome Glyphs have three visual states based on the current zoom level. Additional semantic details are provided at greater zoom levels (see Figure 2 (e)). At the lowest semantic zoom level, the Point Outcome Glyphs only reveal who won the point and whether the point resulted from a good or a bad shot. At the medium semantic zoom level, we add the detail about the specific type of outcome using a single lower-case letter: (a)ce, (w)inner, (f)orced error, (u)nforced error, and (d)ouble fault. At the highest semantic zoom level, we encode point length and sevice information. Point length is encoded using white tick marks designed to look like five-second intervals on a stopwatch. For service information, we use a triangle to encode whether the point started on a first versus second serve (solid black versus hollow white triangle) and service side (deuce $=$ right, ad $=$ left $)$.

Example 2 From Figure 3 (a), we can see a preponderance of bad shots (unforced errors), indicated by hollow balls, in the first set versus the second and third sets. In sets two and three there are more good shots (winners and forced errors), which are indicated by solid balls.

\subsection{Fish Grid View}

The Fish Grid view (see Figure 2(a-d)) provides detailed information about each point within the context of the whole match. A Fish Grid is essentially a $4 \mathrm{X} 4$ matrix where the row indices represent points won by player one and the column indices represent points won by player two. To be consistent with our positioning scheme, this matrix is rotated 45 degrees (resembling a fish) so that all the matrix cells located above the horizontal represent scores where player one is in the lead, cells below the horizontal represent a lead for player two, and cells on the horizontal represent even scores. This maintains the same visual metaphor established for the Pie Meters. Additional cells are added for deuce and ad points, making up the tail of the fish.

If a game goes beyond a single deuce, the tail of the Fish Grid grows to accommodate the extra points, thus making long games readily apparent. For example, in Figure 2 (b), we can see the longest game in the match (20 points). This occurred in the first set with Gonzalez (player 2) serving at 5 games to 6 .

Each cell location in a Fish Grid represents a specific game score (e.g., 30-15). To help users navigate in a grid, a background color is assigned to each cell to indicate whether the score is in favor of player one or player two. In particular, a game win probability (from player one's perspective) for a given score is generated using the same coinflip simulation discussed in Section 4.3. The probabilities are mapped to shades of green (win probabilities $>50 \%$ ), pure white (win probability $=50 \%$ ), and shades of red (win probabilities $<50 \%$ ). Therefore, green cells represent scores in favor of player one while red cells represent scores in favor of player two.

In order to emphasize the scores that actually occurred in the game, only cells corresponding to those scores are colored. In this way, the Fish Grids essentially become sparklines [24], showing the basic temporal trend of each game, even when zoomed out. The vertical position of a Point Outcome Glyph indicates who is currently ahead in the game and its horizontal position encodes point sequence information. For example, Figure 2(a) shows that the game was led by Gonzalez at the very beginning as the result of a winning shot. But this was followed by four good shots in a row by Federer (three winners and a forced error), allowing him to win the game easily. We can also see from the point lengths encoded on the glyphs that both the 0-15 and $40-15$ points started with a first serve and were very short. This indicates that Federer's serves on these points was probably effective. We verified this hypothesis by reviewing the video for these two points.

As with the Pie Meters, the current game score is shown to the left of each Fish Grid and the black ball indicates who is serving. The layout of the games is also the same as in the Pie Meter view.

Users can click on a Point Outcome Glyph in either a Fish Grid or a Pie Meter to play its corresponding match video clip in the video viewer (see Figure 1 (d)) or a standalone window. After watching a video clip, users can manually record their insights associated with the point through the text entry above the video viewer. Points with user observations are highlighted in yellow in both the Pie Meter and Fish Grid views (not shown in the figures). When a user hovers the mouse pointer over a highlighted point, their observations will be presented as a tooltip. When the users reload the video of a point by clicking on its Point Outcome Glyph, the observation will also be displayed in the text entry field above the video viewer so that the user can edit the observation.

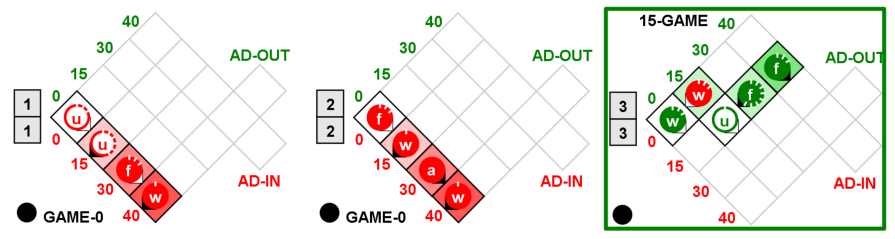

Fig. 5. Fish Grids for games 3, 5, and 7 in the second set showing Gonzalez holding serve easily in games 3 and 5 but then getting broken easily by Federer in game 7 .

Example 3 In examining the second set, shown in Figure 2 (d), we see that in almost all the games, the serving player won easily. The glaring exception is the one and only service break where Federer won four of the five points on Gonzalez' serve. Using the zoom control, we take a closer look at games 3, 5, and 7 in the second set (shown in Figure 5). These show two service games won easily by Gonzalez followed by a service game in which Federer won by a large margin. To understand more closely why Gonzalez was able to win his two service games prior to the service break so easily and then lose the service break game so easily, we click on individual Point Outcome 
Glyphs in the Fish Grid (one at a time) to load them into the video viewer. In examining the videos of the 13 points for these three games, we saw that seven out of the nine points won by Gonzalez started with a serve to Federer's backhand and that all four points won by Federer started with a serve to his forehand.

\subsection{Filters and Bar Charts}

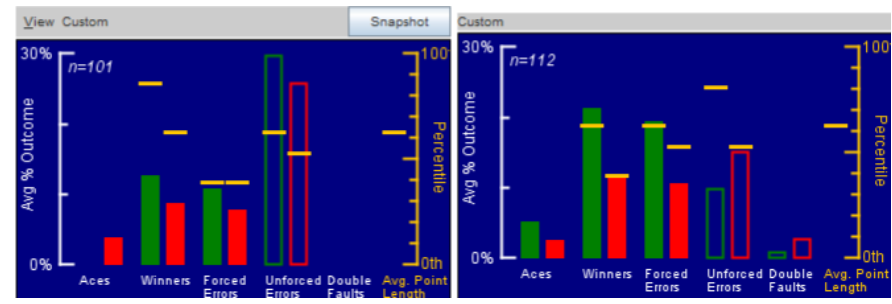

Fig. 6. Bar charts comparing all points in the first set (left) to all points in sets two and three (right). The first set contains a lot of unforced errors and fewer winners and forced errors. Sets two and three have far fewer unforced errors and more winners and forced errors, particularly by Federer (the solid green bars).

The filters (see Figure 1 (a)) allow users to interactively select sets, games and points for further analysis. They can also select an individual player to examine just their shots. When the filters are activated, the unselected games and points in both the Pie Meter and Fish Grid views fade out so that users can focus on the selected ones. Sets are selected by their set numbers. Games can be selected in terms of who is serving and/or who won the game.

A novel game filter called the Game Outcome Filter (see Figure 3 (c)) allows users to distinguish easily won games from close games. Users simply click on one or more pie sectors and only those games whose needle ended within the selected sectors are displayed. Individual points can be selected in terms of who won the point and whether or not the point started from a first or a second serve. There is also a novel pie-shaped filter called the Game Win Probability Filter (shown in Figure 1 (a)) that allows the user to only display points that occurred at specific needle angles (which represent conditional probabilities of winning a game for any given score).

Filters can be turned on and off individually to provide basic insights or used in combination to support various investigative lines of inference. For example, coaches may want to look at all of their player's first serves. To focus on just these points, the coach would select the Serving game filter and the First Serve point filter. To facilitate efficient game analysis (particularly for novice users), a predefined set of common filter configurations is provided in the Filter Configurations Drop-Down (see Figure 1 (a)). The set of predefined filters was added based on feedback from the coaches in our pilot studies. More advanced users can also create, name, and save their own filter configurations.

TenniVis provides a bar chart panel from which users can visually examine the aggregated statistics of outcomes of all points they selected using the filters. In the bar charts (see Figure 6 for examples), the relative percentage of each outcome type (aces, winners, unforced errors, etc.) is displayed. The same visual scheme used to encode information into the Point Outcome Glyphs is used in the design of the bars. Green bars are for points won by player one and red bars for points won by player two. Solid bars refer to good shots and hollow bars refer to bad shots. Users can see the actual number of each type of outcome by hovering the mouse pointer over a bar. A user-selectable secondary Y-axis can also be displayed on the bar charts. Users can choose to view average point lengths or first serve percentages. They can also toggle off this secondary axis completely.

The bar chart panel keeps one or more bar charts side-by-side (see Figure 1 (c)). The leftmost bar chart shows the statistics of the current selection and automatically updates whenever the filters are changed. The other bar charts are snapshots of previous filter configurations. To save a snapshot, users click a button to capture the snapshot for the current filter configuration before they modify the filters to a new configuration. They assign a name to it, which helps when recalling the meaning of the filter configuration. The snapshots enable users to compare a set of point outcomes for one filter configuration with those resulting from different filter configurations. When a new snapshot is created, the vertical scales of all existing bar charts are automatically adjusted to the same to enable comparison. Moreover, users can toggle on a horizontal ruler that spans across all snapshots to compare the bar charts more accurately.

Snapshots also serve for the purpose of insight management. First, clicking on the name of a snapshot brings the filters (and hence the Pie Meter and Fish Grid views) back to the status when the snapshot was captured. Second, observations related to a snapshot can be entered and saved with the snapshot. Third, the snapshot and its related observations are automatically added to the match report for sharing.

Example 4 In the Pie Meter view of the match (see Figure 3 (a)) we noticed a preponderance of bad shots in the first set versus sets two and three. We confirm this by making two bar chart snapshots: one for the first set and one for sets two and three combined (see Figure 6). Comparing these two bar chart snapshots, we see that most of the points in the first set were the result of unforced errors by the players (indicated by the hollow green and red bars). In sets two and three there were far fewer unforced errors and more good shots (winners and forced errors).

Example 5 The Pie Meters in Figure 3 (a) show that there appears to be a majority of short games in favor of Federer. This is easily verified using the Game Outcome Filter (Figure 3 (c)). By selecting the topmost and bottommost pie sectors, we can filter out the close games so we only see games won easily by one player or the other. In doing so, we see that, in Figure 3 (b), Federer won 15 games easily (including the first set tie breaker), while Gonzalez only won four games easily.

Example 6 To finish out our brief analysis of this match, we use the Game Win Probability filter in conjunction with the Game Win filter (see Figure 1 (a)) to identify any "choke" points by the players. A choke point is one where, despite a significant lead in a game by a player, the game is lost. Clicking on the lowest pie sector in the Game Win Probability filter will filter on just those points where Gonzalez was up 40-0 or 40-15. By also clicking on the Game Win filter to only show games won by player one (Federer), we are able to quickly isolate what is perhaps the most critical point in the match. This is shown in the selected point in a Fish Grid from the first set (Figure 2 (c)). This occurred in the first set where Gonzalez was serving at 40-15 with a chance to win the set. At this point in the match, he lost four points in a row, allowing Federer to break his serve. From a game psychology perspective, we see that Federer then won the next game easily on his serve while Gonzalez had to struggle through seven deuces to win his serve. This brought the match to a tie-breaker which was then won easily by Federer.

\subsection{Insight sharing and report generation}

In the previous sections, we saw that users can record insights by annotating points and bar chart snapshots. Therefore, it is straightforward for a user to share his or her insights with others since the annotated video clips and bar chart snapshots are automatically added to a match report. At the present time, only a rudimentary match report is created that consists of a simple HTML page containing the text observations along with their corresponding bar chart snapshot images or links to the associated video clips. In the next iteration of TenniVis, we plan to expand and enhance the report generation capabilities by eliciting feedback from tennis coaches and players concerning what information to include and how to include it effectively.

\section{Pilot User Studies}

To test the effectiveness and usability of the visualizations presented in TenniVis, we conducted two pilot user studies: one with the women's tennis coach and one with the men's tennis coach at a local university. Since TenniVis was designed specifically to be used as an interactive tool for tennis coaches and players, it was important to demonstrate 


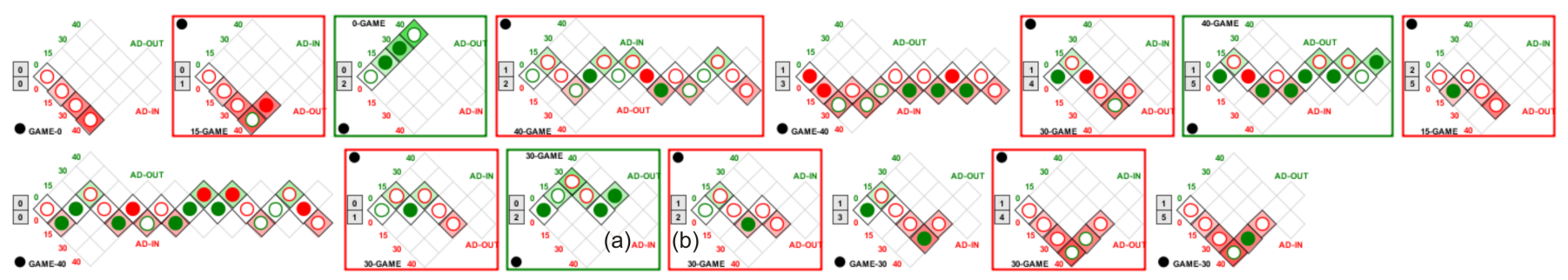

Fig. 7. Fish Grids for a women's singles college match. The coach analyzing this match was particularly interested in games 3 and 4 of the second set $(a, b)$.

that it could be quickly learned by domain experts who don't necessarily have any expertise in visualizations. The goals of these studies were as follows: (1) assess the understandability of visualization graphics (i.e., Pie Meters, Fish Grids, and Bar Charts); (2) assess the perceived efficiency and effectiveness of TenniVis match analysis; and (3) elicit opinions on what type of information and features are needed in the match report (the report generation functions were not in the system during the studies).

\subsection{Procedure}

Using a two-monitor display (24 inch external monitor and a 15 inch laptop monitor), the TenniVis system was setup at the participant's work location in a meeting room. The main TenniVis application was displayed on the external monitor while the laptop monitor was used to display the pop-up video player window. Each pilot study was conducted in three parts: system demonstration, hands-on match analysis, and follow-up questionnaire. After a brief introduction in which the purpose and overview of the study were presented to the participant, the instructor spent 15 minutes demonstrating the various components of TenniVis using the Federer-Gonzalez match. Each of the visualizations and all of the controls were briefly explained to the participant and any questions were answered. The participant was then allowed 10 minutes to explore the system on their own using the same data set.

In the second part of the study, the instructor opened up one of two data sets. For the women's team coach, the data set consisted of a two set singles tennis match of the best singles player on her team recorded a few days earlier (shown in Figure 7). For the men's coach, the data set was a two-set tennis match for the best singles player on his team, also recorded a few days earlier. In both cases, the coaches were present at their respective players' live matches, although they were also watching five other matches simultaneously. The participant was asked to use the TenniVis system to evaluate their player's match and to "think out loud" as they performed this task. Participants then interacted directly with TenniVis as the instructor took notes about which visualizations and components the participants were using and the comments they were making. Since the primary goal of the pilot study was to assess the understandability and usefulness of the visualizations, (and not to assess TenniVis as a finished product) the instructor provided assistance as needed.

After approximately 30 minutes of self-directed interaction with TenniVis, the instructor presented the participant with a brief questionnaire. In this questionnaire, the participant assessed the usefulness and understandability of the overall TenniVis system, the Pie Meters, the Fish Grids, the Point Outcome Glyphs, the Bar Charts, and the video player. Assessments were indicated using Likert scale ratings where users were asked the degree to which they agreed or disagreed with various statements about the TenniVis system. They also had the opportunity to indicate what they liked most about the system, least about the system, and for additional features that would make it more useful. The results of each of these pilot studies are presented next.

\subsection{Pilot Study 1 - Women's Tennis Coach}

When analyzing the women's tennis match, the coach only spent a few minutes on the Pie Meter view (which is the default view when the application is started). She noticed the very high number of service breaks as indicated by the red and green boxes. She then switched to the Fish Grid view (see Figure 7). After scanning through all of the points in order in the Fish Grids, she stopped to more closely examine games three and four in the second set (Figure 7(a) and (b)).

When zoomed into the second semantic zoom level, she was able to see the outcomes of each point in these games (represented by lowercase letters). In game three, she noticed from the Fish Grid how her player (player one) broke her opponent's serve with three solid shots (i.e., two forced errors and one winner) plus an unforced error made by her opponent. She then noted, however, that her player committed two double faults and two unforced errors in her next service game (game four).

Based on prior experience with her player, the participant suspected that her player may have committed the two unforced errors by trying to hit too many down-the-line shots (a potentially risky shot) versus going cross-court. She commented that "I've really been trying to work on her [the player] hitting the ball cross-court more because she goes down the line and then she either misses it or then they make her run cross-court. . . so I was immediately thinking 'How did she lose this point?". She loaded several points one-by-one into the video player and was able to find several examples that confirmed her hypothesis. She then decided to focus on serving and, using the zoom slider, further zoomed into game four to see more details such as first serves vs. second serves. From Figure 7(b), she noticed that five of the six points were from second serves (including two double-faults).

The results of the post-analysis questionnaire indicated that this participant did not fully understand the Pie Meters or the bar charts but found the Fish Grid and video player components very easy to use and understand. The participant commented that she liked the Fish Grid view since it was well organized. We attribute this to the fact that the Fish Grids display all points in the order they occurred and at the game score where they occurred. She indicated that having the supporting evidence provided by the video was useful because, "whenever I ask the girls, 'How do you learn best? ', they always say 'By seeing it; by seeing what you are talking about"'. She said she liked the fact that TenniVis allowed her to directly access video clips for points of interest.

The participant described herself as somewhat averse to technology and indicated that as the primary reason she did not bother to use any of the filters or pay attention to the bar charts. She also had some initial confusion over interpreting the color coding used for the outcome balls, but indicated that having a key would probably solve this problem. Despite some of her issues with specific components of the system, she rated the system overall as easy to understand and use and strongly agreed that it provided her with useful insights that are not currently available to her.

The participant indicated that there is the need to generate simple, compelling reports that communicate her insights in a tangible way her players can readily grasp. She envisioned some type of electronic report that would include her observations along with the supporting video evidence.

\subsection{Pilot Study 2 - Men's Tennis Coach}

The men's tennis coach described himself as a data and statistics junkie with a firm belief in the power of statistics and analysis to be used as a 


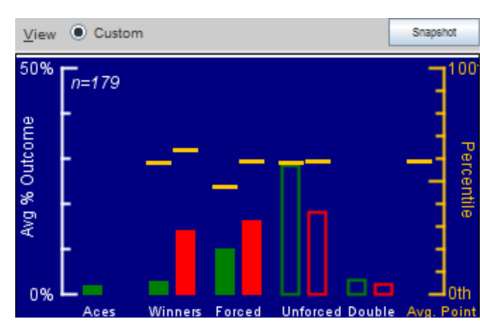

(a)

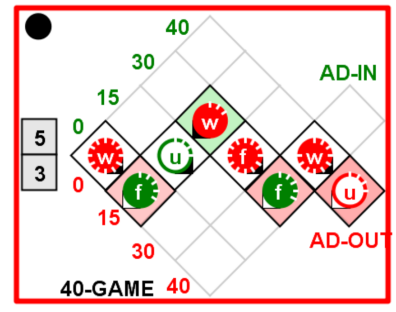

(b)
Fig. 8. (a) Histogram of a men's singles college match. The coach noticed that, even though his player (player one) made fewer unforced errors (hollow red bar) than the opponent (hollow green bar), these were not enough to make up for the large number of winners and forced errors made by the opponent (red solid bars) versus his player (green solid bars). (b) Eighth game in the second set showing a critical point at 30-0 where the player setup his opponent for a winner.

tool to complement his training program. He was able to quickly grasp the meaning of the color coding used in the Pie Meters and for the Point Outcome Glyphs, commenting "the color coding is nice...the red and green is good because that's intuitive". In looking at the Pie Meters from the Federer-Gonzalez match, he commented "it looks like right off the bat he [Federer] was dictating play... because he has a lot of solid green points which means he's winning a lot of points" (as opposed to getting points due to bad shots from Gonzalez). Attention was then turned to the Fish Grid view where the participant commented that "you see kind of trends, up and down... and you see what the momentum looks like".

Once the data set for his own player was brought up, he immediately looked at the histogram (see Figure 8 (a)) coupled with the Pie Meter view to get an overall sense of the difference between the two players in terms of point outcomes. He commented that "overall, their guy hit certainly more winners... he also had more forced errors and our guy had less unforced errors... our guy doubled less and had a few more aces... but that looks a little negligible in terms of risk/reward". He then began using various filter combinations to create individual histogram snapshots for comparison, noticing that his player had a lot of points that started from his second serve.

Continuing his analysis after switching to the Fish Grid view, he noticed a key game in set two when his player was serving at five games to three (see Figure 8 (b)). He focused in on the point when his player was up 30-15 (only two points away from winning the set) and his opponent hit a winner (see Figure 8 (b)). The opponent then was able to regain the upper hand in the match and ultimately win it. $\mathrm{He}$ analyzed why his player lost this point by selecting it to view in the video player. He noticed that, although his player served his opponent with a tough serve out wide, he failed to capitalize on this advantage and gave his opponent an easy putaway shot at the net. This insight led the coach to indicate he would discuss shot selection with his player to avoid giving away the momentum in a match.

The results of the post-analysis questionnaire indicated the participant found the system to be very useful and easy to understand. He agreed strongly that it gave him useful match insights he is not currently able to get. He commented that, with the system, he can look at trends and get a visual sense of what was going on. The Pie Meter, Fish Grid, and video player components got very high marks for usefulness. The participant indicated that, while he liked the ability to animate the Pie Meter needles to "see what was going on", he preferred the Fish Grid over the Pie Meter because it was like a timeline that presented the ups and downs.

Both the Point Outcome Glyphs and the histograms got neutral usefulness ratings. The participant explained that the reason for the lower usefulness score for the Point Outcome Glyphs was that he really would like to see if the outcome was from a backhand or forehand. This issue can be addressed by collecting the backhand/forehand information in data collection and encoding it in the Point Outcome Glyphs.
He gave several useful suggestions on improving the usability of the histograms. First, he suggested allowing users to manually select games to be displayed in a histogram, rather than just relying on the filters. Second, he suggested a set of pre-defined filter configurations representing some of the standard statistics, such as service games, first serves, etc. Third, he recommended displaying actual numbers of outcomes instead of just percentages. These features were not in the system during the pilot user studies, but were added later based on this feedback. He indicated that giving his players actual numbers instead of just percentages would resonate more with them. Overall, the participant commented that he liked the fact that there are multiple ways to visualize the data in TenniVis.

The participant also confirmed the need for generating reports to communicate his insights to his players. He envisioned being able to use this system and then generate a report he could tape to a player's locker that provided specific insights that player needed to focus on.

\section{Conclusion AND Future Work}

In this paper, we present TenniVis, a novel tennis visualization system. It demonstrates how visualizations useful for tennis coaches and players can be generated using only the easily collected non-spatial game data and a video from a consumer level camera. Two new visual metaphors, namely the Pie Meter and the Fish Grid, are proposed and implemented in TenniVis. They convey summary and detailed information about a match in an organized way that is understandable by users with tennis domain expertise. The multi-resolution visualization pipeline, dynamic query capabilities, and ad hoc hypothesis development and testing capabilities provided by TenniVis make it an effective tool for tennis match analysis.

Through two pilot user studies, we verified that tennis coaches were quickly able to gain insights into their players' tennis matches through TenniVis and found the visualizations easy to use and understand. With only a minimal amount of training, coaches were able to interact with TenniVis to confirm suspicions they already had about a tennis match and to find new insights. They were excited about the prospect of having a tool to help them share their observations with their players. One of the coaches actually contacted us after the study to request a copy of the Fish Grid view for her player.

While we have demonstrated the utility of TenniVis to analyze individual matches, we plan to expand it to be able to visualize multiple matches in order to see longer term trends. This may also then lead to the need to view multiple video segments simultaneously. For example, a coach may want to view specific video segments before a coaching intervention is made and then after to see if the advice given was followed by a player. We plan on working with coaches to determine how to most effectively capture their analysis findings and communicate them to their players. We also plan to work with players to determine their ability to grasp the meaning of the visualizations and to see if they can find useful insights in their own games.

We acknowledge the insightful benefits provided by systems relying on tracking data and see our efforts as complementary to them. In the future, we would like to extend TenniVis for professional players by integrating the visual analytics of tracking data. In addition, we would also like to explore whether the visualizations in TenniVis can be applied to other sports such as table tennis and badminton, since they have structures similar to tennis.

Acknowledgements We would like to thank Daniel Keim for his timely suggestions on improving the visualizations presented in this paper. We would also like to thank the reviewers for their highly constructive feedback that allowed us to fill several key gaps. This work is partially supported by National Science Foundation under grant numbers IIS-1352893 and IIS-1352927.

\section{REFERENCES}

[1] Dartfish sports analysis software. http://www.dartfish.com/, 2013.

[2] Information about a tennis court from Wikipedia. http://en.wikipedia.org/wiki/Tennis_court/, 2013.

[3] MotionView Tennis Video Analysis Software. http://www.tenniscoachsystems.com/, 2013. 
[4] ProTracker Tennis Software. http://www.protrackertennis.com/, 2013.

[5] IBM SlamTracker used at the 2014 Australian Open. http://www.ausopen.com/en_AU/slamtracker/, 2014.

[6] USTA Friend at Court 2014. http://www.itatennis.com/, 2014.

[7] C. Ahlberg, C. Williamson, and B. Shneiderman. Dynamic queries for information exploration: An implementation and evaluation. In Proceedings of the SIGCHI conference on Human factors in computing systems, pages 619-626. ACM, 1992.

[8] R. Borgo, J. Kehrer, D. H. Chung, E. Maguire, R. S. Laramee, H. Hauser, M. Ward, and M. Chen. Glyph-based visualization: Foundations, design guidelines, techniques and applications. In Eurographics 2013-State of the Art Reports, pages 39-63. The Eurographics Association, 2012.

[9] C. Ó. Conaire, P. Kelly, D. Connaghan, and N. E. O'Connor. Tennissense: A platform for extracting semantic information from multi-camera tennis data. In Digital Signal Processing, 2009 16th International Conference on, pages 1-6. IEEE, 2009.

[10] D. Connaghan, P. Kelly, and N. E. O'Connor. Game, shot and match: Event-based indexing of tennis. In Content-Based Multimedia Indexing (CBMI), 2011 9th International Workshop on, pages 97-102. IEEE, 2011.

[11] A. Cox and J. Stasko. Sportsvis: Discovering meaning in sports statistics through information visualization. In Compendium of Symposium on Information Visualization, pages 114-115, 2006.

[12] K. Goldsberry. Courtvision: New visual and spatial analytics for the nba. MIT Sloan Sports Analytics Conference, 2012.

[13] L. Jin and D. C. Banks. Visualizing a tennis match. In Information Visualization'96, Proceedings IEEE Symposium on, pages 108-114. IEEE, 1996.

[14] P. A. Legg, D. H. Chung, M. L. Parry, M. W. Jones, R. Long, I. W. Griffiths, and M. Chen. Matchpad: Interactive glyph-based visualization for real-time sports performance analysis. In Computer Graphics Forum, volume 31, pages 1255-1264. Wiley Online Library, 2012.

[15] J. Mackinlay. Automating the design of graphical presentations of relational information. ACM Transactions on Graphics (TOG), 5(2):110$141,1986$.

[16] J. Muthukumarasamy and J. T. Stasko. Visualising Program Executions on Large Data Sets Using Semantic Zooming. Graphics, Visualization \& Usability Center, College of Computing, Georgia Institute of Technology, Technical Report GIT-GVU-95-02, 1995.

[17] N. Owens, C. Harris, and C. Stennett. Hawk-eye tennis system. In IEE conference publication, pages 182-185. Institution of Electrical Engineers, 2003.

[18] C. Perin, R. Vuillemot, and J.-D. Fekete. Soccerstories: A kick-off for visual soccer analysis. Visualization and Computer Graphics, IEEE Transactions on, 19(12):2506-2515, 2013.

[19] H. Pileggi, C. D. Stolper, J. M. Boyle, and J. T. Stasko. Snapshot: Visualization to propel ice hockey analytics. Visualization and Computer Graphics, IEEE Transactions on, 18(12):2819-2828, 2012.

[20] G. Pingali, A. Opalach, and Y. Jean. Ball tracking and virtual replays for innovative tennis broadcasts. In Pattern Recognition, 2000. Proceedings. 15th International Conference on, volume 4, pages 152-156. IEEE, 2000.

[21] G. Pingali, A. Opalach, Y. Jean, and I. Carlbom. Visualization of sports using motion trajectories: providing insights into performance, style, and strategy. In Proceedings of the conference on Visualization'01, pages 7582. IEEE Computer Society, 2001.

[22] G. S. Pingali, Y. Jean, and I. Carlbom. Lucentvision: A system for enhanced sports viewing. In Visual Information and Information Systems, pages 691-698. Springer, 1999.

[23] C. Plaisant, J. Grosjean, and B. B. Bederson. Spacetree: Supporting exploration in large node link tree, design evolution and empirical evaluation. In Information Visualization, 2002. INFOVIS 2002. IEEE Symposium on, pages 57-64. IEEE, 2002.

[24] E. Tufte. Sparklines: Intense, simple. Word-Sized Graphics, 2004.

[25] M. O. Ward. A taxonomy of glyph placement strategies for multidimensional data visualization. Information Visualization, 1(3-4):194-210, 2002.

[26] M. O. Ward. Multivariate data glyphs: Principles and practice. In Handbook of Data Visualization, pages 179-198. Springer, 2008.

[27] C. Williamson and B. Shneiderman. The dynamic homefinder: Evaluating dynamic queries in a real-estate information exploration system. In Proceedings of the 15th annual international ACM SIGIR conference on Research and development in information retrieval, pages 338-346. ACM, 1992.

[28] G. Zhu, C. Xu, Q. Huang, W. Gao, and L. Xing. Player action recognition in broadcast tennis video with applications to semantic analysis of sports game. In Proceedings of the 14th annual ACM international conference on Multimedia, pages 431-440. ACM, 2006. 